\title{
Potential avenues of linking the energy efficiency and the sustainable economic development in the Balkan region
}

\author{
Jasmina Mangafić ${ }^{1 *}$, Ognjen Riđić ${ }^{2}, J_{0 s i p}$ Nikolić $^{3}$, Azra Smječanin ${ }^{4}$ \\ ${ }^{1}$ School of Economics and Business, University of Sarajevo, Bosnia \\ ${ }^{2}$ Faculty of Business and Administration, International University of Sarajevo, Bosnia \\ ${ }^{3,4}$ Ministry of Spatial Planning of the Federation entity of BiH, Bosnia
}

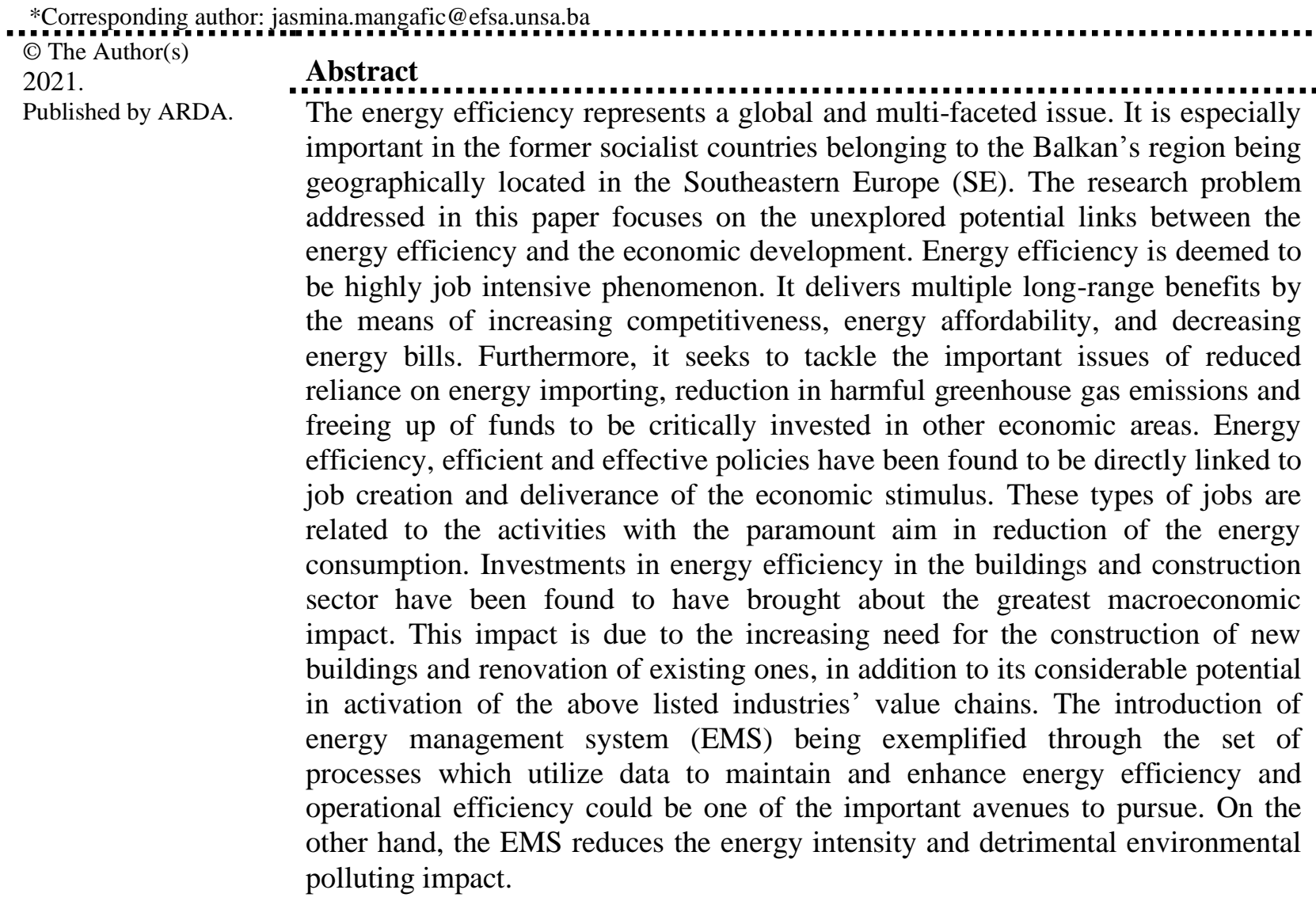

Keywords: Energy efficiency, Sustainable economic development, Energy management system (EMS), Balkan region

\section{Introduction}

The energy efficiency represents one of the critical resources for the social and the economic development. This is especially true for the former socialist countries belonging to the Balkan's region of the south-eastern Europe. The energy efficiency captures the primary place as a major energy resource in the context of national and international activities to achieve sustainability metrics. As such, it represents a paradigm shift representing, both, the supply and demand side in achievement of the economic growth. Governments and related institutions are facing an increased pressure in order to enhance their energy consumption, which has

This work is licensed under a Creative Commons Attribution License (https://creativecommons.org/licenses/by/4.0/) that allows others to share and adapt the material for any purpose (even commercially), in any medium with an acknowledgement of the work's authorship and initial publication in this journal. 
been largely regarded as a fundamental component in the sustainable economic development. Energy efficiency has been singled out as the most cost-effective method addressing the challenges of achieving the sustainable economic growth. These actions are being supported by the means of enhancement of the energy security, competitiveness and the environmental sustainability. Four strategic considerations have been crystallized to aid policymakers in bringing about the most efficient and most effective economic stimulus. First of all, the energy efficiency endeavors can be linked to supporting of the economic stimulus programs through support of existing labor force and creation of the new jobs. Second aspect involves government facilitation of better economic results by utilization of large-scale investment programs. Third aspect involves the importance of the market considerations focusing on high energy efficiency and setting of incentives. Finally, the fourth aspect addresses the need for government to deliver the economic stimulus packages by utilizing scale and speed. This includes the standardization of designs, eligibility criteria in contract, innovation in retrofitting and technology upgrades all the way to the philosophy, as to how the energy efficiency can be included into all-encompassing government stimulus packages. The policy makers in developed economies have grasped the sense for cities (urban areas) to focus significant part of its economic development efforts on energy management in the public residential buildings. By doing so, they tend to attract and keep valued businesses with their jobs and tax revenues. The linkage between the energy efficiency and energy management efforts, in order to enhance the economic development has shown the growth strategy with two elements. First element is being exemplified through the so-called carrot of energy competition, while the second element focuses on benchmarking and disclosure requirements [1], [2], [3], [4].

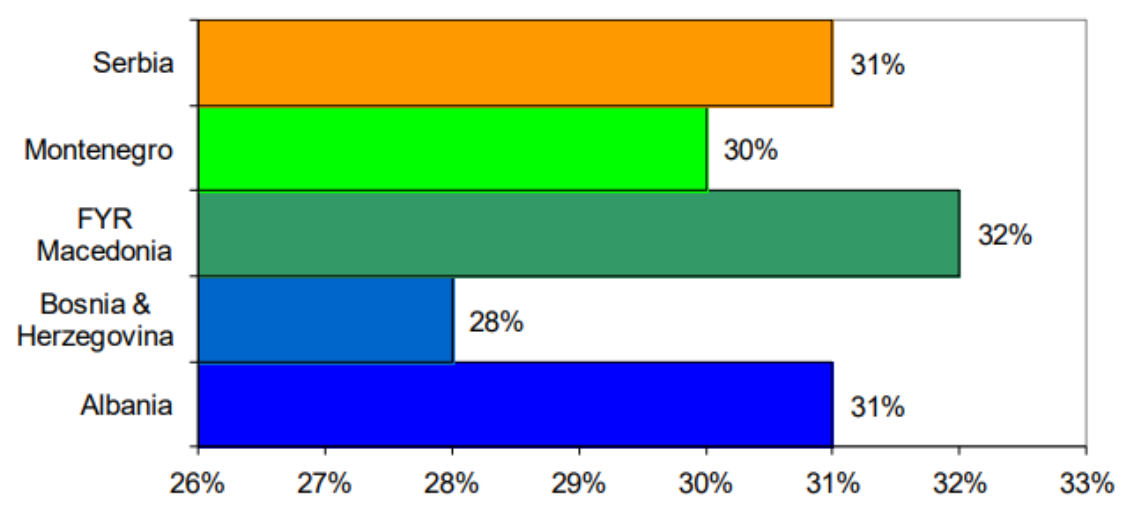

Figure 1. The energy consumption in Western Balkan's (WB's) region Residential Sector [5]

As Fig. 1 above shows, the energy consumption in WB's residential sector was the largest in North Macedonia with 32 percent and the lowest in Bosnia and Herzegovina (BiH) with 28 percent.

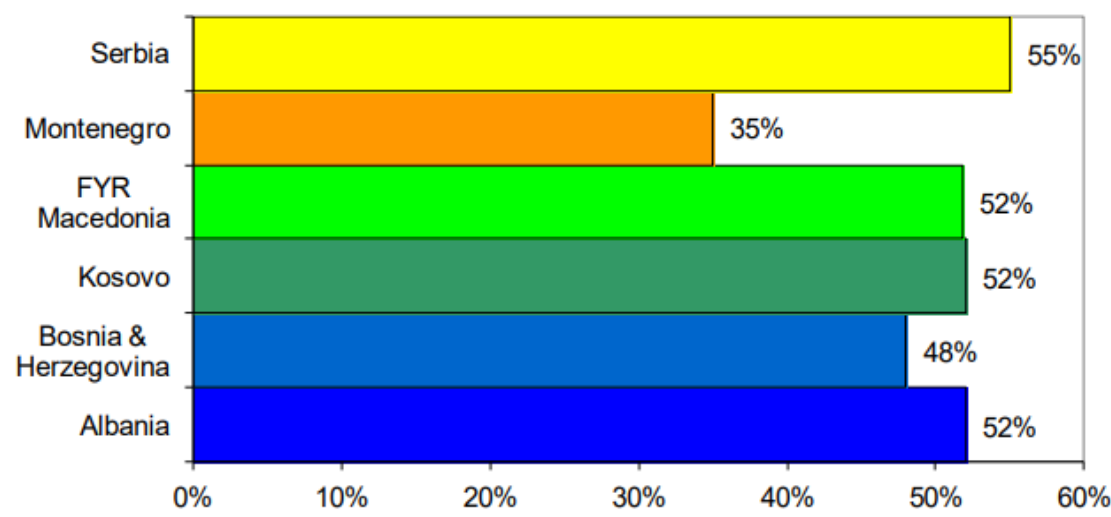

Figure 2. The share of electrical energy consumption in Western Balkan's region [5]

Per Fig. 2, displayed above, the largest share of electrical consumption energy in WB's region was in Serbia with 55 percent and the lowest in $\mathrm{BiH}$ with 48 percent, respectively. 


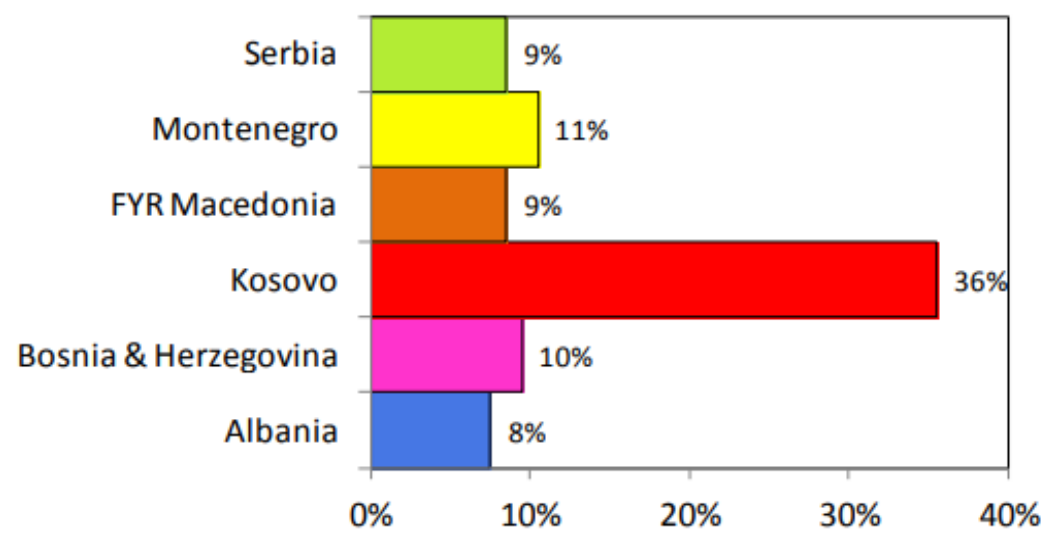

Figure 3. The share of energy subsidies as percentage of the Gross Domestic Product (GDP) for the Western Balkan's region [5]

As depicted in the Fig. 3, above, the largest share of energy subsidies as the percentage of GDP in the WB's region was by far in Kosovo with 36 percent, the lowest in Albania with 8 percent, while $\mathrm{BiH}$ averaged 10 percent.

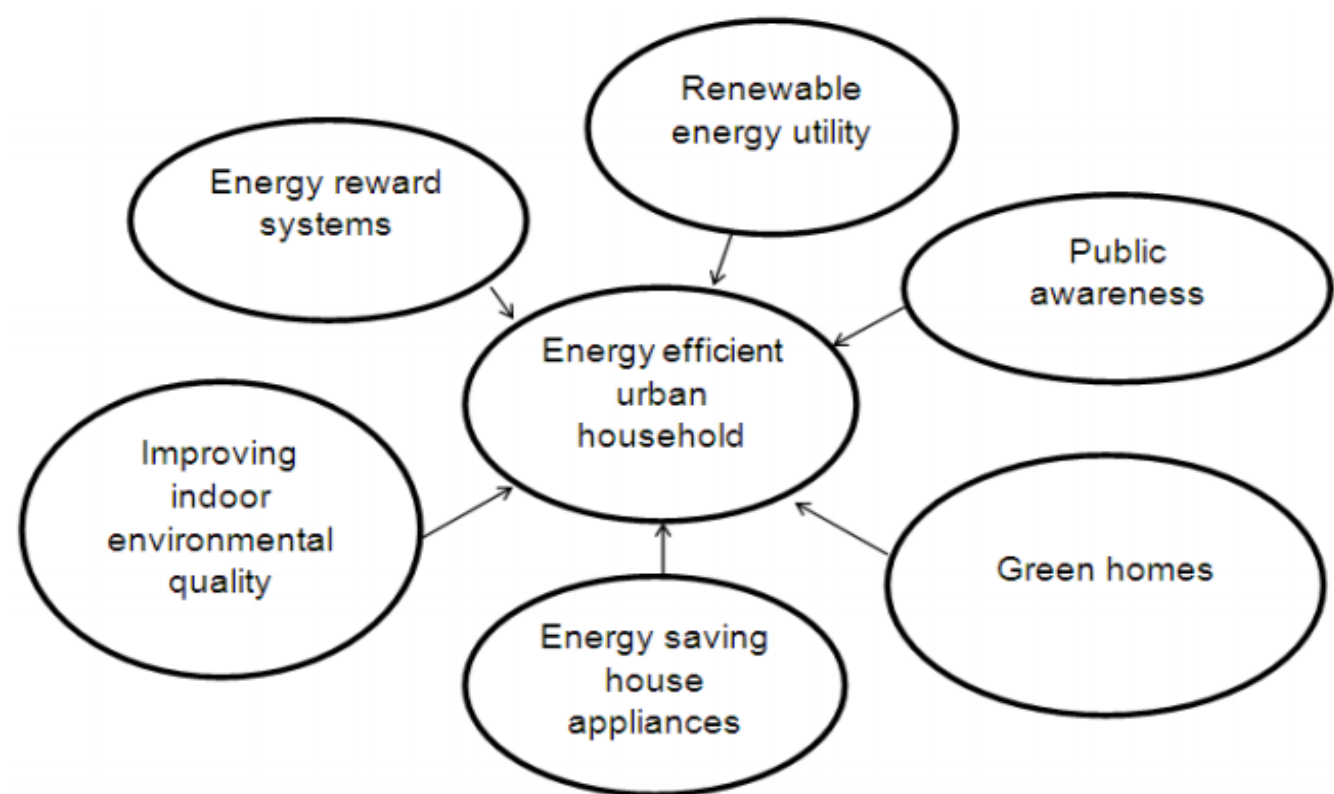

Figure 4. Energy efficiency strategies for urban residential stock [4]

Furthermore, Ganda \& Ngwakve [4] expanded the carrot and stick approach to the improvement of the energy efficiency in the light of its contribution to the sustainable economic development by including six contributory elements to the energy efficient open household. Per Fig. 4, above, these elements are being exemplified in the green homes, energy saving house appliances, improving indoor environmental quality, energy reward systems, renewable energy utility and an increased public awareness.

Nunez-Ferrer [6] depicted the Revolving Fund model, in the Fig. 5, above. It shows the centrally placed revolving fund, which receives private equity financing in addition to the state guarantees in the forms of loans, promotional banks, and other types of funds. In continuation, the revolving fund is being fed by favorable types of funding provided by the banking financial institutions.

\section{Materials and methods}

This research paper utilizes detailed review of multi-faceted resources (peer-reviewed journal articles related to the environmental economics, various governments', European Union (EU)), European Commission (EC), International Energy Agency (IAE) and private publications)) in order to discern the answers to the outlined research problem. 


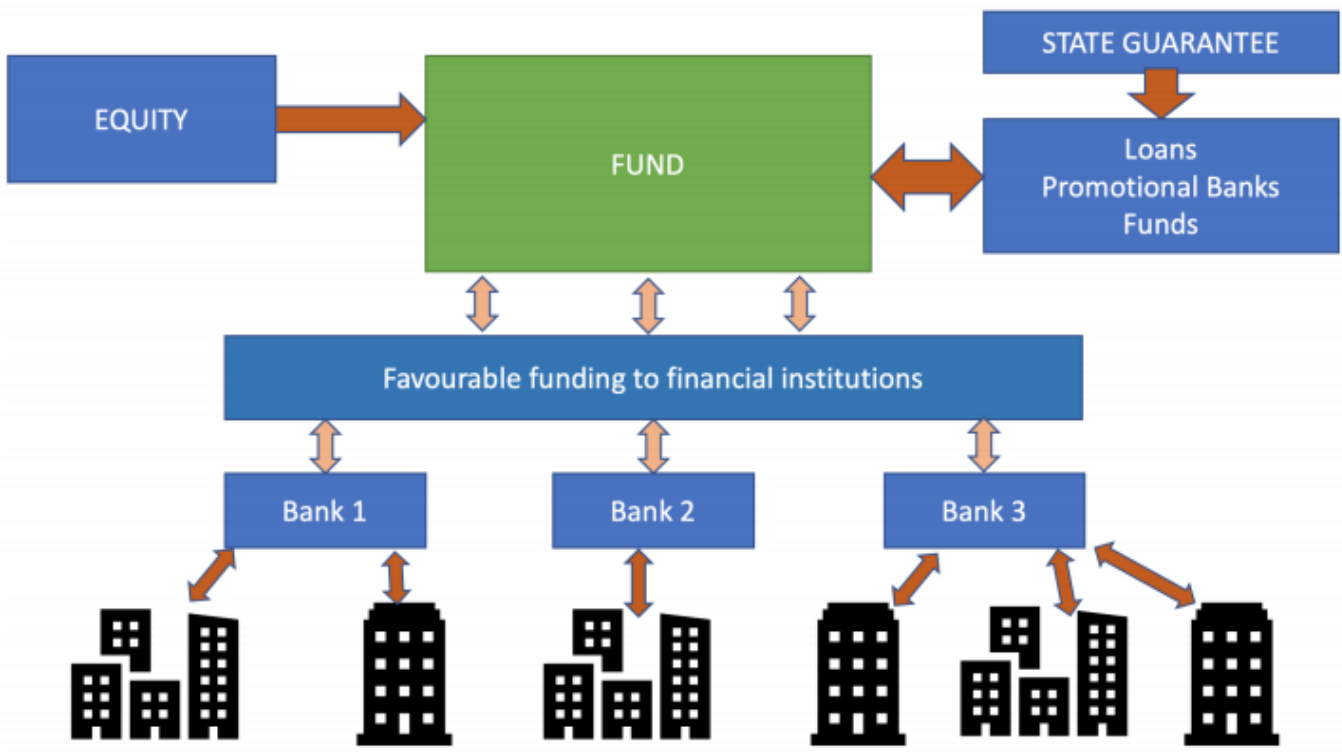

Figure 5. Revolving fund model with associated funding flows [6]

\section{Results and discussion}

The significant opportunities of linking energy efficiency efforts and stimulating the economy have been identified in the residential housing sector. For example, they may include programs focused to the new buildings and the upgrade programs for the existing buildings. New building programs revolve around the focus on construction to meet high energy efficiency standards. Reliance on cost effective solutions shall deliver numerous benefits. If they are implemented on a large scale by inclusion of innovations, they can result in significant cost reductions an improved efficiency. On the other hand, improving programs focusing on upgrades look into the provision of incentives to enable the energy efficient upgrades to the residential buildings at scale. Almost two-thirds of the aggregate expenditures on home energy efficiency retrofitting tend to be devoted to labor related costs, which in turn augment the employment growth. Public sector programs aimed at standardized building types, such as hospitals, office buildings, schools, and social housing have proven its effectiveness in creating numerous and considerable economic benefits. The above discussed incentives can be either utilized from the already present programs or can be created from the scratch, in order to advance the economic stimulus and job creation [3], [7], [8].

Governments, at various levels, tend to offer the incentives directly to consumers in order to replace the old and inefficient products, such as household appliances and motor vehicles. Furthermore, the replacement programs can act as the stimulus to the employment, while creating the utilization of more efficient devices. Technology replacement programs can assist the manufacturing sector by bringing to the market newer and more efficient technologies, such as digital building management systems, the electric vehicles, heat pumps and cooling/ventilating/air-conditioning units (HVAC). Other aspects of technology replacement initiatives may include fleet upgrade initiatives related to the motor vehicles, such as trains, buses, transportation vans and taxis. The infrastructures projects can create higher number of jobs by utilizing, both, the public procurement and local value chains. They are focusing on investments, which enable energy efficiency. Some of these examples are smart grids and charging of the electric vehicles, next generation digital connectivity for energy generation systems and the upgrades in public street lightning. In India, second most populous country in the worlds its "Street lightning national program" manage to upgrade more than 11 million streetlights generating 13,000 jobs and reducing greenhouse gas emissions by 5,000,000.00 tons, annually [3], [7].

As far as the infrastructure projects are concerned the economic stimulus programs, worldwide, will have to involve increased investment levels in the buildings and infrastructure. Furthermore, the construction of high efficiency residential block should decrease the greenhouse gas emissions by $50 \%$ over the life of the building. It is important to note that this involves minimal construction related costs in comparison with the construction of energy inefficient buildings. Residing in a high efficiency residential block unit can significantly reduce the energy related bills. The reduction in these kinds of bills can ultimately free up saved monies to another economic activities, such as investments in the financial instruments or education. When discussing the importance of energy efficiency inclusion in all stimulus investments it is critical to consider, which avenues are the best to target the public funding in addition to the availability of the private capital. It is 
also important to note that the public residential stock homeowners will be largely unwilling to spend their savings or to take the loans to make home upgrades if there is a lack of affective public financial support programs [3], [7].

Non-sustainable human energy practices have contributed to the destruction of natural environments in addition to an increased pollution. These detrimental practices are linked with harmful climate changes caused by the global warming, droughts, desert expansion and diseases [3], [7], [8].

\section{Conclusion}

In conclusion this article compares and contrast some avenues and policy mechanisms that can be considered to create more effective policies linking energy efficiency efforts and the economic growth and development. The grants and rebates, cash for replacement of old and inefficient vehicles, programs delivered by energy utilities, green procurement, bulk procurement, and direct installation programs. The critical analysis of the effects of energy efficiency on the sustainable economic development can in the aggregate be viewed in the light of carbon reduction, detrimental effects of climate change, and the unemployment and poverty reduction. As far as policy approaches are concerned a critical look into the energy efficiency and the economic stimulus programs conducted by the governments in the past could provide for valuable insights into the improvements of program designs and implementation.

\section{References}

[1] J. Spencer, "The Growing Link Between Energy Efficiency and Economic Development", pp. 1-2, September 4, 2015.

[2] OECD/IEA, "Capturing the Multiple Benefits of Energy Efficiency: Executive Summar", pp. 18-25, 2014.

[3] The European Commission, "Financing Energy Efficiency in Central and South-Eastern Europe," Executive Agency for Small and Medium-sized Enterprises (EASME), 28 June 2018, pp. 3-6, 2018.

[4] K. Ganda, C.C. Ngwakwe, "Role of energy efficiency on sustainable development", Environmental Economics., vol. 5., no. 1 pp. 86-98, 2014.

[5] B. Kalkum, "Scaling Up Energy Efficiency in Buildings in Western Balkans": Financing Energy Efficiency Measures for Residential Building Stock", Guidance Note 89324, World Bank Group, pp. 428, May 2014.

[6] J. N. Ferrer, "Leveraging funding for energy efficiency in buildings in South Eastern Europe" in CEPS Policy Insights - Thinking Ahead for Europe, No 2019-05/28 March 2019.

[7] F. O. Ulusoy and E. Pektaş, "Recent trends and issues in energy conservation technologies", Heritage and Sustainable Development, vol. 1, no. 1, pp. 33-40, 2019.

[8] S. T. Satir and N. Dogan-Saglamtimur, "Proficiency of Maritime English course: An investigation in Istanbul, Turkey", Heritage and Sustainable Development, vol. 3, no. 1, pp. 6-15, Jan. 2021. 